\title{
Balance and gait performance after maximal and submaximal endurance exercise in seniors: is there a higher fall-risk?
}

\author{
Lars Donath • Lukas Zahner • Ralf Roth • \\ Livia Fricker • Mareike Cordes • Henner Hanssen · \\ Arno Schmidt-Trucksäss • Oliver Faude
}

Received: 4 January 2012/ Accepted: 27 July 2012 / Published online: 23 August 2012

(C) Springer-Verlag 2012

\begin{abstract}
Impaired balance and gait performance increase fall-risk in seniors. Acute effects of different exercise bouts on gait and balance were not yet addressed. Therefore, 19 healthy seniors (10 women, 9 men, age: $64.6 \pm 3.2$ years) were examined on 3 days. After exhaustive treadmill testing, participants randomly completed a $2-\mathrm{km}$ treadmill walking test $\left(76 \pm 8 \% V_{2 \max }\right)$ and a resting control condition. Standing balance performance (SBALP) was assessed by single limb-eyes opened (SLEO) and double limb-eyes closed (DLEC) stance. Gait parameters were collected at comfortable walking velocity. A condition $\times$ time interaction of center of pressure path length $\left(\mathrm{COP}_{\text {path }}\right)$ was observed for both balance tasks $(p<0.001)$. Small (Cohen's $d=0.42, p=0.05)$ and large $(d=1.04, p<0.001) \mathrm{COP}_{\text {path }}$ increases were found after 2-km and maximal exercise during DLEC. Regarding SLEO, slightly increased $\mathrm{COP}_{\text {path }}$ occurred after 2-km walking $(d=0.29, p=0.65)$ and large increases after exhaustive exercise $(d=1.24, p<0.001)$. No significant differences were found for gait parameters. Alterations of SBALP after exhaustive exercise might lead to higher fallrisk in seniors. Balance changes upon 2-km testing might be of minor relevance. Gait is not affected during single task walking at given velocities.
\end{abstract}

Keywords Upright stance · Gait variability · Elderly · Older · Physical activity $\cdot$ Exercise training

Communicated by Fausto Baldissera.

L. Donath $(\square) \cdot$ L. Zahner · R. Roth · L. Fricker · M. Cordes · H. Hanssen - A. Schmidt-Trucksäss · O. Faude

Institute of Exercise and Health Sciences, University of Basel, Birsstrasse 320B, 4052 Basel, Switzerland

e-mail: lars.donath@unibas.ch

\section{Introduction}

Increasing life expectancy seriously affects worldwide population aging and proportions. Despite regional disparities, the global percentage of the population aged $\geq 60$ years was expected to increase from 10 to $32 \%$ until the end of the present century (Lutz et al. 2008). With $46 \%$ in the year 2100 , the highest percentage of this agegroup was estimated for Western Europe (Lutz et al. 2008). This apparent societal over-aging is even more critical against the background of health-care utilization and expenditures. In this regard, unintentional falls and its related injuries are considered to be major economic and public health burdens in the elderly. Depending on morbidity status, the annual fall rates of seniors aged $\geq 65$ years range between 0.3 and 1.6 falls (weighted mean 0.6) per person (Rubenstein 2006) and the associated direct medical care costs amounts to $\$ 0.2$ billion for fatal and $\$ 19$ billion for nonfatal fall-related injuries (Stevens et al. 2006). Studies addressing circumstances and mechanisms that lead to a higher fall-risk within the process of aging are therefore mandatorily required.

Beside a variety of reported extrinsic fall-risk factors (e.g. lack of handrail, uneven terrain, twilight, obstacles), a body of evidence emphasized that aging-associated personal factors (intrinsic risk factors), such as (a) visual impairment, (b) medication intake, (c) strength deficits of the lower limb, (d) gait variability and (e) impaired balance performance should be obligatorily taken into account in terms of reducing the risk of falling in older people (de Menezes and Bachion 2008; Granacher et al. 2010a, 2011a; Kressig et al. 2008).

Several studies investigated the long-term influence of physical exercise interventions on intrinsic fall-risk factors, such as e.g. strength, balance and gait performance training 
in older people (Hauer et al. 2001, 2006). It has been shown, for example, that music-based multitask training (Trombetti et al. 2011) and balance as well as strength training can significantly improve both fall-risk predicting parameters (Granacher et al. 2011b). However, acute effects of exercise bouts on these fall-risk factors are rarely examined (Parijat and Lockhart 2008; Helbostad et al. 2007, 2010a). These studies predominantly reported fatigue-induced alterations of balance and gait performance due to repeated sit-to-stand tasks or various resistance exercises. Data on acute influences of maximal and submaximal aerobic exercise on balance and gait performance are not available to date. This is particularly interesting since such exercises are often prescribed for cardiovascular endurance training in the elderly or they are exposed to similar demands in everyday life.

Therefore, the present study was conducted to investigate the acute effects of a single bout of (1) exhaustive maximal endurance exercise and (2) submaximal exercise that is common during everyday life on single and double limb standing balance performance (SBALP) as well as temporal and spatial gait parameters. It was hypothesized that exercise-induced alteration of standing balance and gait performance might lead to a higher susceptibility to sustain falls in the elderly. These results might contribute to a better understanding of higher fall risks during everyday life and following certain regimes of aerobic exercise in the older and aging population.

\section{Methods}

\section{Participants}

Based on the physical activity readiness questionnaire (PAR-Q), medical history was taken and physical examination was performed in 19 active seniors (Table 1), who took part in the present study. All participants did not report any medication intake and relevant health impairments, such as orthopedic, neurological and internal diseases that may affect standing balance and gait performance. Moreover, all participants had to successfully complete a 12-lead electrocardiogram (ECG) during rest and maximal exercise. The participants were additionally asked to refrain from any severe exercise within the last $72 \mathrm{~h}$ prior to the examination days. The study was approved by the local ethics committee (Ethikkommission beider Basel, EKBB, Basel, Switzerland) and complied with the Declaration of Helsinki. All participants signed an informed written consent prior to the start of the study.
Study design

The present study was conducted as semi-randomized controlled cross-over trial. All participants were examined on 3 days in weekly intervals. On each day, measurements were intra-individually performed at the same time of day. Due to the required exercise-ECG (Custo cardio 100, Custo med GmbH, Ottobrunn, Germany), the first day was obligatorily set as maximal exercise test. Before starting maximal exercise testing, the "Freiburg physical activity questionnaire" (Frey et al. 1999) and the "Fall efficacy scale" (FES-I, validated German version) (Helbostad et al. 2010b) were applied in order to obtain secondary physical activity and fear of falling data. On the second and third day, either the submaximal 2-km walking test or the resting control condition was randomly conducted. Balance and gait testings were conducted before and after each interventional condition.

Interventions

\section{Maximal exercise test}

On the first day, a maximal exhaustive ramp-like exercise test on a treadmill (h/p Cosmos, Pulsar 4.0, HP-Cosmos Sports \& Medical GmbH, Nussdorf-Traunstein, Germany) was performed. A well-established age-adapted exercise protocol was applied. This walking-based "Pepper-protocol" combines the increase of inclination (\%) and velocity (miles per hour) and is described in detail elsewhere (Peterson et al. 2003). Briefly, this exercise protocol started with an inclination of $0 \%$ and a velocity of $1.5 \mathrm{~m}$ per hour. Exercise intensity was increased every minute by elevating either treadmill inclination or velocity until subjective maximal exhaustion level was reported. After reaching objective spiroergometric exhaustion levels in order to obtain maximal oxygen uptake (heart rate above age-predicted maximum, respiratory exchange ratio (RER): $>1.15$ ) (Midgley et al. 2007), subjects immediately underwent standing balance and gait testing.

\section{Submaximal 2-km exercise test}

In accordance to Oja et al. (1991), a submaximal 2-km walking test was conducted on the same treadmill of the maximal exercise test. The corresponding velocity and inclination of the CR-10 Borg-value of "four" during maximal exercise testing was used to determine a moderate exercise intensity for the $2-\mathrm{km}$ exercise test. The subjects were requested to perform a total distance of $2 \mathrm{~km}$ at this exercise intensity. 
Table 1 Anthropometric, physical activity and exercise data of the participants

\begin{tabular}{|c|c|c|c|}
\hline & Women $(n=10)$ & Men $(n=9)$ & Total $(n=19)$ \\
\hline \multicolumn{4}{|l|}{ Personal data } \\
\hline Age (years) & $64.6 \pm 3.7$ & $64.6 \pm 2.7$ & $64.6 \pm 3.2$ \\
\hline Weight (kg) & $63.2 \pm 10.1$ & $76.6 \pm 7.8$ & $69.6 \pm 11.2$ \\
\hline Height (m) & $1.62 \pm 0.05$ & $1.79 \pm 0.05$ & $1.70 \pm 0.10$ \\
\hline BMI $\left(\mathrm{kg} \mathrm{m}^{-2}\right)$ & $24.1 \pm 3.5$ & $23.9 \pm 2.6$ & $24.0 \pm 3.0$ \\
\hline Physical activity (h Woche ${ }^{-1}$ ) & $12.5 \pm 6.0$ & $9.2 \pm 5.0$ & $11.0 \pm 5.5$ \\
\hline FES-I & $16.4 \pm 0.7$ & $16.3 \pm 0.5$ & $16.4 \pm 0.6$ \\
\hline \multicolumn{4}{|l|}{ Maximal ramp-test } \\
\hline$t(\min )$ & $22.7 \pm 2.1$ & $22.9 \pm 3.7$ & $22.8 \pm 2.9$ \\
\hline$V \mathrm{O}_{2 \max }\left(\mathrm{L} \mathrm{min}^{-1} \mathrm{~kg}^{-1}\right)$ & $32.4 \pm 4.1$ & $30.1 \pm 5.8$ & $31.3 \pm 4.5$ \\
\hline $\mathrm{HR}_{\max }\left(\min ^{-1}\right)$ & $168 \pm 8$ & $163 \pm 11$ & $165 \pm 10$ \\
\hline RER & $1.19 \pm 0.10$ & $1.29 \pm 0.08$ & $1.24 \pm 0.10$ \\
\hline RPE & $9.4 \pm 1.0$ & $9.7 \pm 0.5$ & $9.5 \pm 0.8$ \\
\hline \multicolumn{4}{|l|}{ Submaximal $2-\mathrm{km}$ walking test } \\
\hline$t(\min )$ & $28.5 \pm 4.0$ & $26.8 \pm 3.1$ & $27.7 \pm 3.6$ \\
\hline$V \mathrm{O}_{2}\left(\mathrm{~L} \mathrm{~min}^{-1} \mathrm{~kg}^{-1}\right)$ & $23.5 \pm 4.9$ & $24.9 \pm 4.4$ & $24.2 \pm 4.6$ \\
\hline$V \mathrm{O}_{2 \max }(\%)$ & $72 \pm 7$ & $77 \pm 5$ & $75 \pm 6$ \\
\hline $\operatorname{HR}\left(\min ^{-1}\right)$ & $131 \pm 8$ & $124 \pm 9$ & $127 \pm 10$ \\
\hline RER & $0.90 \pm 0.08$ & $0.97 \pm 0.09$ & $0.94 \pm 0.09$ \\
\hline RPE & $3.7 \pm 0.8$ & $4.3 \pm 0.9$ & $4.0 \pm 0.9$ \\
\hline $\mathrm{BR}\left(\min ^{-1}\right)$ & $30 \pm 5$ & $28 \pm 7$ & $29 \pm 6$ \\
\hline
\end{tabular}

$B M I$ body mass index, physical activity derived from the Freiburger physical activity questionnaire, FES-I sum score of the validated German version of fall efficacy scale questionnaire, $t$ exercise time during the maximal ramp-like exercise test, $V O_{2 \max }$ oxygen uptake, $H R$ heart rate, $R E R$ respiratory exchange ratio, $R P E$ subjectively perceived exertion level at the Borg CR-10 scale, Borg averaged perceived exertion level during the 2-km walking test, $B R$ breathing rate; data are provided as mean $\pm \mathrm{SD}$

\section{Resting control condition}

To provide an individual control measure, patients underwent a resting control condition in standardized supine posture without any postural demands or other distraction. They wore comfortable clothes, but no sport wear. The examination room was kept silent, with comfortable temperature between 21 and $24^{\circ} \mathrm{C}$. In order to provide a feasible and inter-individually objective and standardized real resting condition with reduced potential influences of any interfering external and internal effects during sitting or standing, we have chosen the supine position. Thus, subjects remain in a supine position within the similar timeframe $(\sim 30 \mathrm{~min})$ of the performed or estimated $2-\mathrm{km}$ exercise time, respectively.

\section{Testing procedure}

\section{Standing balance parameters}

Following a 5-min familiarization period before premeasurements by trying all relevant standing balance positions (two times, $10 \mathrm{~s}$ for each condition), the testing procedure was started immediately after a short postfamiliarization break of $3 \mathrm{~min}$. The piezoelectric Kistler ${ }^{\circledR}$ force platform (KIS) was used to assess SBALP and installed on a flat and rigid laboratory floor according to the manufacturer's installation requirements. Compared to strain gauge-basing systems, piezoelectric measures produce a very small signal drift. Despite a drifting signal, piezoelectric force plates are, however, regarded as suitable in terms of force-plate basing standing balance measures (Middleton et al. 1999). In addition, such a potential but negligible bias would occur during all conditions and tasks and, thus, it seems unlikely that the present results would be affected. Laboratory light ( $\sim 500$ luces $)$, temperature $\left(21^{\circ} \mathrm{C}\right)$ and air humidity $(60 \%)$ were kept stable. The order of measured standing balance tasks was double limb stance with closed eyes (DLEC) first and second, single limb stance with open eyes (SLEO). The dominant leg for SLEO was determined via the lateral preference inventory (Coren 1993). Three attempts for each standing balance task were performed. Both standing balance tasks were tested for $30 \mathrm{~s}$, followed by a post-exercise break of $1 \mathrm{~min}$. 
The participants were instructed in a standardized manner to (a) perform without shoes, (b) place their feet comfortably, nearly parallel at approximately shoulder-width on the platform, (c) place the hands on the hips, (d) bend the knees slightly and (e) stand as still as possible while focusing a marked circle at the nearby wall (distance: $1.5 \mathrm{~m}$; height: $1.75 \mathrm{~m}$ ).

\section{Gait parameters}

Before starting the gait analysis, the individual comfortable walking velocity was measured using two photoelectric timing gates (Tag Heuer, HL 2-31, la Chaux-de-Fonds, Switzerland). Therefore, all participants were instructed to walk a total distance of $12 \mathrm{~m}$ in an even hallway for three times. 10 of the total $12 \mathrm{~m}(1-11 \mathrm{~m})$ were measured in order to calculate the average walking velocity. To mirror everyday walking conditions, subjects were requested to walk normally (like going for a walk), without rushing, acceleration or deceleration. Each subject had one free trial. Then, the average velocity of the three consecutive walking attempts was applied on a one-dimensional ground reaction force measuring treadmill (Zebris Medical $\mathrm{GmbH}$, FDM-T system, Isny, Germany) for gait analysis. This treadmill was different from the one used during maximal and submaximal exercise testing. After a short familiarization period of $1 \mathrm{~min}, 10 \%$ below the target velocity and one additional minute at the target velocity, data acquisition started. Subjects had to walk 400 steps on the treadmill at their a priori evaluated comfortable walking velocity.

\section{Secondary outcome variables}

Heart rates (Accurex Plus, Polar Electro-Oy, Kempele, Finland) and gas exchange data were collected during maximal and submaximal exercise testing in order to achieve (a) objective maximal exhaustion level and (b) a controlled submaximal ventilatory and cardiac exercise response during the $2-\mathrm{km}$ walking test.

\section{Data acquisition and analysis}

\section{Standing balance measurements}

Standing balance data were collected within a time frame of $30 \mathrm{~s}$ using a frequently applied sampling rate in postural control measures of $40 \mathrm{~Hz}$ (Granacher et al. 2010b). As single limb balance tasks-in contrast to the bipedal stance-are usually performed for $10 \mathrm{~s}$ (Clark et al. 2010), we calculated center of pressure (COP) path lengths for a 10 -s interval between the 5th and 15th second of the total $30 \mathrm{~s}$ data acquisition time. To avoid high frequency noise contamination, a system-immanent and commonly employed Butterworth filter with a low pass cut-off frequency of $10 \mathrm{~Hz}$ was applied to the time-related signals (Salavati et al. 2009) of the KIS ground reaction forces. Total center of pressure (COP) path length serves as outcome measure, which has been frequently applied to determine SBALP (Clark et al. 2010; Salavati et al. 2009). Out of the three consecutive repetitions for both standing balance tasks, the mean of the two shortest COP path lengths of the three attempts were included into statistical analyses.

\section{Gait analysis}

Temporal and spatial gait parameters, such as mean stride frequency (steps/min), stride width $(\mathrm{cm})$, double stride time (seconds) and length $(\mathrm{cm})$ as well as double stance length were obtained during 400 steps of comfortable walking velocity. The relevant gait parameters were averaged over the required 400 walking steps (Owings and Grabiner 2003). Gait variability data were calculated using the coefficient of variances $(\mathrm{CoV})$ for temporal and spatial stride-to-stride length and time. Both parameters were indicated as percentage values.

\section{Maximal and submaximal ventilatory and heart rate} data

Spiroergometric data were collected using a breath-by breath spirometric system (Metamax 3B, Cortex, Leipzig, Germany). These data were averaged to provide data points for each $10 \mathrm{~s}$ time-interval. The mean of the highest three consecutive $V \mathrm{O}_{2}$ values within a 30-s time frame of the final exercise step was regarded as maximal oxygen uptake $\left(V \mathrm{O}_{2 \max }\right)$. Additionally, the highest recorded heart rate, RER, and perceived exertion level (Borg-scale, CR-10) were considered as maximal exercise values. These data were recorded to provide a standardized and objective maximal physical exhaustion level during maximal exercise testing. Percentages of $V \mathrm{O}_{2 \max }$ and $\mathrm{HR}_{\max }$ were given to indicate the ventilatory and heart rate response during the submaximal 2-km Borg-paced walking test.

Statistical analysis

First, data were tested for normal distribution (Kolmogorov-Smirnov Test) as well as homogeneity of variances (Levene Test) and were provided as mean and standard deviations (SD). All dependent parameters were analyzed by two (SLEO, DLEC) separately conducted 2 (time: pre, post) $\times 3$ (repeating factors, condition: maximal exercise, submaximal exercise, control condition) repeated measure analysis of variances (rANOVA). Follow-up Tukey HSD 
post hoc tests were separately conducted in case of a significant main condition effect or condition $\times$ time interaction.

Effect sizes (Cohen's $d$, trivial: $d<0.2$, small: $0.2 \leq d<0.5$, moderate: $0.5 \leq d<0.8$, large $d \geq 0.8$; Cohen 1992) and the percentage changes of the COP path length displacements between pre- and post-measures for maximal exercise, submaximal exercise and the control condition during SLEO and DLEC were additionally calculated. Statistical significance level was set at $p<0.05$.

\section{Results}

Anthropometric and exercise performance data

All data are presented in detail in Table 1. Both groups consist of homogeneous distributed male and female subjects. No gender differences were found regarding anthropometric and physiological variables. Included subjects did not report fear of falling, were physically active and not overweighted. Maximal exercise parameters indicated good physical fitness (relative oxygen uptake) and objective exhaustion occurred during maximal exercise testing. Submaximal exercise was performed at moderate perceived exertion and around $75 \%$ of maximal oxygen uptake.

\section{Static SBALP}

Significant condition $\times$ time interactions were observed both for SLEO $(F=29.7, p<0.001)$ and DLEC $(F=23.2, p<0.001)$. However, post hoc testing solely revealed significant differences of COP path length between pre and post testing following maximal endurance exercise for SLEO and DLEC (Fig. 1). Submaximal exercise did not affect both standing tasks. Merely the supine control condition leads to decreased path length during SLEO. Moreover, a significant time-effect for SLEO $(F=31.8, p<0.001)$ and DLEC $(F=51.1, p<0.001)$ was found. Detailed means and SDs with its significance levels are presented in Table 2.

Regarding percentage changes and effect size calculation of postural sway alterations between pre and post testing, small decreases of $\mathrm{COP}_{\text {path }}$ were found at the control day (DLEC: $-8 \%$, effect size $(d)=0.21$, $p=0.60$; SLEO: $-22 \%, d=0.47, p=0.04)$. In turn, small (sub: $+18 \%$, Cohen's $d=0.42, p=0.05$ ) as well as large ( $\max :+52 \%, d=1.04, p<0.001)$ increases of $\mathrm{COP}_{\text {path }}$ were found following submaximal $2 \mathrm{~km}$ and maximal walking during DLEC. Similarly during SLEO, slightly increased $\mathrm{COP}_{\text {path }}$ were found after the $2-\mathrm{km}$ walking test $(\sim+15 \%, d=0.29, p=0.65)$ and large increases after exhaustive exercise $(\sim+88 \%, d=1.24$, $p<0.001)$.

Temporal and spatial gait analysis data

A significant time $\times$ condition interaction was merely found for double stance phase $(p=0.004)$. However, post hoc testing did not reveal significant differences between pre and post testing. The detailed rANOVA results including means and differences are provided in Table 3.

\section{Discussion}

To our knowledge for the first time, the present study revealed evidence that maximal and submaximal endurance exercise bouts affect SBALP during single and double limb stance in population of healthy and active seniors. Compared to the relatively small alterations of postural sway following submaximal $2-\mathrm{km}$ exercise testing, large effect sizes and percentage changes of COP path length displacements were observed after maximal exhaustive walking exercise. In contrast, no relevant changes of temporal and spatial gait parameters were observed within the 400 steps of treadmill gait analysis at individually given walking velocities and under single task condition for all three testing conditions.

The observed elevations of COP path length displacements, especially pronounced during single limb stance, suggest that at least healthy and active seniors showed elevated fall-risk parameters following exhaustive physical exercise. Beside exercise-induced alterations of respiration and otolith function that may influence postural control (Schmid et al. 2004; Charles et al. 2002), also central and peripheral fatigue processes might contribute to this finding (Helbostad et al. 2010a). However, we did not objectively measure neuromuscular fatigue, by e.g. EMG activity patterns, in order to address this issue with certainty. With respect to comparatively alterations of postural sway, previous findings in adults revealed similar percentage changes and effect sizes upon maximal exercise in adults (Gauchard et al. 2002) and younger subjects (Nardone et al. 1997). Nevertheless, baseline COP path length data have been shown to be more elevated in seniors (Abrahamova and Hlavacka 2008). It can be additionally assumed that prolonged walking with repetitive eccentric components may lead to alterations of proprioception, potentially by alterations of mechanoreceptors function (Riskowski et al. 2005) and an overstimulation of the otolith function (Lepers et al. 1997; Charles et al. 2002).

Although maximal exhaustive exercise bouts with similar high exercise intensities are less common during daily life in much older, co-morbid or even frail seniors, higher 


\section{maximal ramp-test submaximal walking control condition}

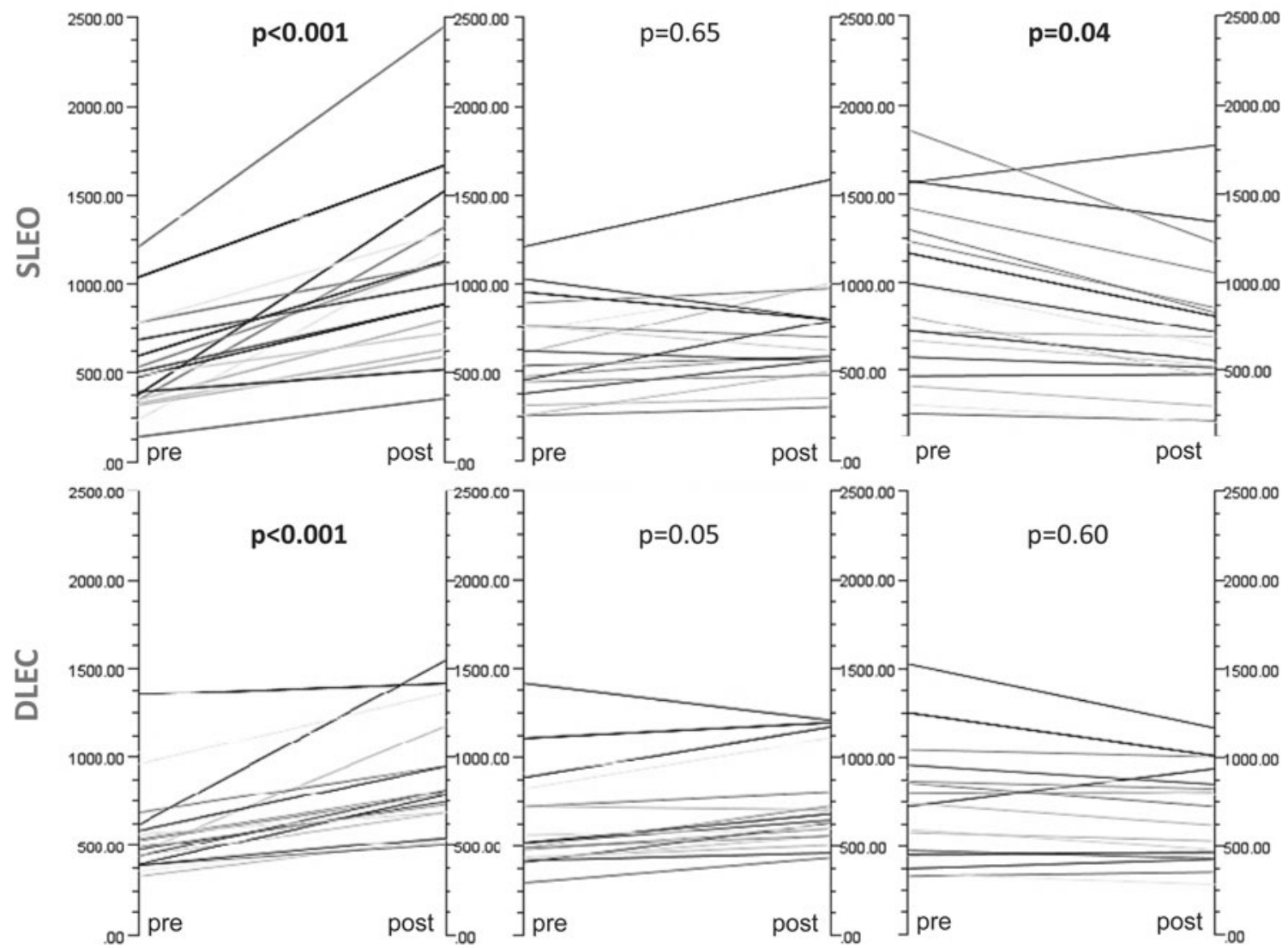

Fig. 1 Single data line-plots between pre and post testing during single limb stance-opened eyes (SLEO, upper three charts) and double limb stance-eyes closed (DLEC, lower three charts) for the maximal ramp-like exercise test (a), submaximal 2-km walking test (b) and control condition (c). $P$ values of the Tukey HSD post hoc tests are provided for each chart. Significant level was set at $p<0.001^{* * *} ; p<0.01^{* * *}$ and $p<0.05^{*}$
Table 2 Pre and post center of pressure (COP) path length displacements $(\mathrm{mm})$ after maximal exercise, submaximal 2-km testing and control condition during single limbeyes open (SLEO) and double limb-eyes closed (DLEC) upright stance

\begin{tabular}{llrl}
\hline & \multicolumn{1}{l}{ Pre } & \multicolumn{1}{l}{ Post } & Cohen's $d$ effect size \\
\hline SLEO & & & \\
Maximal & $577 \pm 332^{* * *}$ & $1,083 \pm 482$ & +1.24 \\
Submaximal & $661 \pm 314$ & $761 \pm 368$ & +0.29 \\
Control & $961 \pm 466^{*}$ & $754 \pm 408$ & -0.47 \\
DLEC & & & \\
Maximal & $582 \pm 263^{* * *}$ & $884 \pm 317$ & +1.04 \\
Submaximal & $640 \pm 282^{*}$ & $754 \pm 263$ & +0.42 \\
Control & $746 \pm 320$ & $685 \pm 254$ & -0.21 \\
\hline
\end{tabular}

exercise intensities might occur at least in active and sportive seniors, e.g. when hiking, rushing to the bus or train, or during specific high-intensity exercise programs. High intensity exercise training applying several short bouts of nearly maximal individual intensity (e.g. 4 times, 4 min at $95 \%$ of maximal heart rate) has been increasingly considered to be beneficial in various clinical and nonclinical populations (Kemi and Wisloff 2010). Considering these contexts, also higher exercise intensities seem reasonable in this certain group of fit and healthy seniors. Independent of seniors' fitness and health state, an appropriate time of post-exercise recovery should then be warranted in order to avoid immediate exercise- or fatigueassociated falls. This recommendation is of greater importance since younger athletes need 15 post-exercise minutes to sufficiently recover to baseline performance of 
Table 3 Temporal and spatial gait parameters for the three conditions (maximal, submaximal, control) during pre and post testing

\begin{tabular}{|c|c|c|c|c|c|c|c|c|c|}
\hline \multirow{2}{*}{$\begin{array}{l}\text { Gait } \\
\text { parameter }\end{array}$} & \multicolumn{2}{|c|}{ Maximal exercise test } & \multicolumn{2}{|c|}{ Submaximal $2-\mathrm{km}$ test } & \multicolumn{2}{|c|}{ Control condition } & \multicolumn{3}{|c|}{ rANOVA } \\
\hline & Pre & Post & Pre & Post & Pre & Post & $\begin{array}{l}\text { Time } \\
(p)\end{array}$ & $\begin{array}{l}\text { Condition } \\
(p)\end{array}$ & $\begin{array}{l}\text { Condition } \times \\
\text { time }(p)\end{array}$ \\
\hline $\begin{array}{l}\text { Stride frequency } \\
\left(\text { steps } \min ^{-1}\right)\end{array}$ & $62.1 \pm 4.3$ & $61.8 \pm 4.4$ & $61.3 \pm 5.3$ & $60.1 \pm 4.6$ & $60.1 \pm 5.5$ & $60.1 \pm 5.0$ & 0.237 & 0.104 & 0.484 \\
\hline Stride width $(\mathrm{cm})$ & $8.7 \pm 2.6$ & $8.3 \pm 2.8$ & $8.9 \pm 2.5$ & $8.6 \pm 2.5$ & $8.8 \pm 2.4$ & $8.7 \pm 2.5$ & 0.031 & 0.162 & 0.394 \\
\hline Double stride time (s) & $0.97 \pm 0.07$ & $0.98 \pm 0.07$ & $0.99 \pm 0.09$ & $1.00 \pm 0.09$ & $0.99 \pm 0.09$ & $0.99 \pm 0.08$ & 0.421 & 0.058 & 0.418 \\
\hline Double stride length $(\mathrm{cm})$ & $132.1 \pm 12.5$ & $131.4 \pm 12.7$ & $134.5 \pm 12.1$ & $135.1 \pm 11.4$ & $134.8 \pm 12.1$ & $134.5 \pm 11.0$ & 0.793 & $0.001^{\mathrm{a}}$ & 0.663 \\
\hline Temporal gait variability $(\%)$ & $1.9 \pm 0.010$ & $1.8 \pm 0.8$ & $1.5 \pm 0.7$ & $1.4 \pm 0.4$ & $1.4 \pm 0.4$ & $1.4 \pm 0.4$ & 0.301 & $0.001^{\mathrm{a}}$ & 0.782 \\
\hline Spatial gait variability $(\%)$ & $2.4 \pm 1.1$ & $2.4 \pm 1.0$ & $2.2 \pm 1.0$ & $2.0 \pm 1.0$ & $2.0 \pm 1.0$ & $2.0 \pm 1.0$ & 0.804 & $0.009^{\mathrm{a}}$ & 0.223 \\
\hline Double stance phase $(\%)$ & $25.0 \pm 2.2$ & $24.7 \pm 2.3$ & $24.4 \pm 2.9$ & $24.5 \pm 2.5$ & $24.2 \pm 2.5$ & $24.5 \pm 2.5$ & 0.906 & 0.329 & $0.004^{b}$ \\
\hline
\end{tabular}

Data are provided as mean \pm standard deviation. Temporal and spatial gait variability data were presented as percentage values of coefficient of variance (CoV) data of double stride time (temporal) and double stride length (spatial)

${ }^{a}$ Post hoc testing revealed differences between maximal and submaximal as well as maximal and control

b Post hoc testing did not reveal significance differences for any condition and time

postural control (Fox et al. 2008). Unfortunately, it is not clear in which time-frame older adults are able recover from exercise-induced alterations of SBALP to baseline COP path length displacements. Further studies are needed to address this issue with repeated measure time-series analyses of standing balance parameters after exercise in the elderly.

Alterations of SBALP following submaximal endurance exercise bouts have been found to be less pronounced in seniors. Differences concerning single limb stance between pre and post testing were not within statistical significance. Percentage changes between pre and post testings were less than $20 \%$ and the calculated effect sizes were merely moderate. Thus, these changes seem to be of minor practical relevance.

Regarding potential standing balance training approaches, it appears reasonable to assume that visual impairment might additionally increase exercise-induced alterations of postural control since the process of aging results in a more vision-based sensory feedback during upright standing balance tasks (Colledge et al. 1994). In accordance to findings of Chapman and Hollands, it might be paid attention on applying open-eyed dynamic balance tasks pre-dominantly to improve the role of vision during feed forward mechanisms, for example, random goal-orientated stepping tasks (Chapman and Hollands 2006a). This more obstacle-orientated approach might also be beneficial since older adults particularly need to look earlier ahead to future stepping targets and fix them for a longer period of time to place their feet accurately (Chapman and Hollands 2006b).

With respect to the gait analysis, we did not find relevant alterations of temporal and spatial gait parameters after a submaximal and maximal bout of endurance exercise. Despite an condition $\times$ time interaction of the double stance phase and condition effects of double stride length and temporal as well as spatial gait variability, we did not find post hoc differences between pre and post testing following both exercise regimes. Interestingly, the observed condition effects might be addressed to a familiarization effect since the maximal exercise test was always conducted first. Generally, walking at given velocities and under single task condition appears as a relative stable and rigid pattern. However, further studies should address fatigue-induced gait alterations (a) under dual- and multitask condition, (b) during gait analysis without given velocities on a treadmill and (c) in older, less active and frailer seniors with notably higher fall efficacy scores.

Some limitations need to be addressed concerning the present study. We solely investigated healthy, active and less frail seniors without any health impairments. Further cross-sectional and prospective studies are required in more fall-prone and fragile older seniors with additional health impairments. Compared to the included subjects, it seems plausible that seniors with more cardiovascular or cardiocirculatory comorbidities will not achieve such maximal exercise intensities during everyday life. It seems merely likely that larger alteration of postural sway and temporal and spatial gait parameters may occur even following submaximal exercise regimes in this population. Moreover, the present results show merely general response of static and dynamic postural control following exercise in an active and physically fit population of seniors and, thus, should not interpreted interchangeably with comorbid subject suffering from any age-related diseases. Healthy and fit elderly are still able to achieve exhaustive exercise intensities and participate in sport programs. From a health-care perspective, also these subjects are relevant. The present data also does not allow comparative age-related conclusions of deteriorations of 
postural sway following both exercise regimes. We cannot address with certainty whether the amount of these particular endurance exercise-induced alterations of balance performance will be similarly occur in young adults or seniors suffering from any cardiac, metabolic or neurological disease that frequently occur in seniors. Concerning gait analysis, we did not examine gait parameters during normal gait at different velocities. Treadmill walking is known to represent a kind of artificial walking. Also investigations addressing the recovery time frame to baseline COP path length displacements are needed in order to estimate the "open-window" of an exerciseinduced higher risk of falling. Such recovery time-frame data are, however, available at least in young adults (Nardone et al. 1997). Moreover, the study was merely semi-randomized since the first day has to be set as an exhaustive exercise-ECG in order to exclude any adverse exercise-induced cardiac events. The control condition was performed without shoes and at the same intra-individual time of day, but with normal street clothes. This fact might explain the systematically elevated baseline path length at the control day. This assumption underpins the finding of Heller and colleagues, who reported elevated path length when wearing a bag pack ( 15-20\% of bodyweight). Slightly heavier clothes (jeans, sweater) may also lead to prolonged COP path lengths. Despite decreasing COP path lengths during the control day might suggest a learning effect, we would assume that the more sedative and recovering supine position during the control day might lead to a higher parasympathical drive, which in turn may induced an even stiller and quieter upright stance. However, further research would be needed to address this issue with certainty.

In conclusion, SBALP is notably affected following maximal aerobic endurance exercise of a total duration between 20 and $30 \mathrm{~min}$ in active and healthy seniors. Following a submaximal everyday-life endurance exercise, for example, walking or shopping, or an aerobic exercise training session seniors showed slightly impaired static SBALP. Dynamic balance during gait is not relevantly affected in this age-group. To more thoroughly address changes in fall-risk factors as a consequence of submaximal endurance exercise, further studies with asymmetric loads, different walking distances and intensities seem to be warranted in young adults, seniors with and without common comorbidities and risk factors, respectively, such as smoking (including COPD), obesity, diabetes, high blood pressure or cardiac disease (CHD, CHF).

Acknowledgments The authors would like to appreciate the engagement of the subjects during the study. Additional thank is dedicated to the ProSenectute society (Basel, Switzerland) who supported the acquisition of the included subjects.
Conflict of interest None declared.

\section{References}

Abrahamova D, Hlavacka F (2008) Age-related changes of human balance during quiet stance. Physiol Res 57(6):957-964

Chapman GJ, Hollands MA (2006a) Age-related differences in stepping performance during step cycle-related removal of vision. Exp Brain Res 174(4):613-621

Chapman GJ, Hollands MA (2006b) Evidence for a link between changes to gaze behaviour and risk of falling in older adults during adaptive locomotion. Gait Posture 24(3):288-294

Charles C, Cian C, Nougier V, Bigard XA, Job A, Raphel C (2002) Over-stimulation of the vestibular system and body balance. J Vestib Res 12(2-3):135-143

Clark RA, Bryant AL, Pua Y, McCrory P, Bennell K, Hunt M (2010) Validity and reliability of the Nintendo Wii Balance Board for assessment of standing balance. Gait Posture 31(3):307-310

Cohen J (1992) A power primer. Psychol Bull 112(1):155-159

Colledge NR, Cantley P, Peaston I, Brash H, Lewis S, Wilson JA (1994) Ageing and balance: the measurement of spontaneous sway by posturography. Gerontology 40(5):273-278

Coren S (1993) The lateral preference inventory for measurement of handedness, footedness, eyedness, and earedness: norms for young adults. Bull Psychon Soc 31(1):1-3

de Menezes RL, Bachion MM (2008) Study of intrinsic risk factors for falls in institutionalized elderly people. Cien Saude Colet 13(4):1209-1218

Fox ZG, Mihalik JP, Blackburn JT, Battaglini CL, Guskiewicz KM (2008) Return of postural control to baseline after anaerobic and aerobic exercise protocols. J Athl Train 43(5):456-463

Frey I, Berg A, Grathwohl D, Keul J (1999) Freiburg questionnaire of physical activity-development, evaluation and application. Soz Praventivmed 44(2):55-64

Gauchard GC, Gangloff P, Vouriot A, Mallie JP, Perrin PP (2002) Effects of exercise-induced fatigue with and without hydration on static postural control in adult human subjects. Int J Neurosci 112(10):1191-1206

Granacher U, Gruber M, Gollhofer A (2010a) Force production capacity and functional reflex activity in young and elderly men. Aging Clin Exp Res 22(5-6):374-382

Granacher U, Iten N, Roth R, Gollhofer A (2010b) Slackline training for balance and strength promotion. Int $\mathrm{J}$ Sports Med 31(10):717-723

Granacher U, Bridenbaugh SA, Muehlbauer T, Wehrle A, Kressig RW (2011a) Age-related effects on postural control under multitask conditions. Gerontology 57(3):247-255

Granacher U, Muehlbauer T, Zahner L, Gollhofer A, Kressig RW (2011b) Comparison of traditional and recent approaches in the promotion of balance and strength in older adults. Sports Med 41(5):377-400

Hauer K, Rost B, Rutschle K, Opitz H, Specht N, Bartsch P, Oster P, Schlierf G (2001) Exercise training for rehabilitation and secondary prevention of falls in geriatric patients with a history of injurious falls. J Am Geriatr Soc 49(1):10-20

Hauer K, Becker C, Lindemann U, Beyer N (2006) Effectiveness of physical training on motor performance and fall prevention in cognitively impaired older persons: a systematic review. Am J Phys Med Rehabil 85(10):847-857

Helbostad JL, Leirfall S, Moe-Nilssen R, Sletvold O (2007) Physical fatigue affects gait characteristics in older persons. J Gerontol A Biol Sci Med Sci 62(9):1010-1015 
Helbostad JL, Sturnieks DL, Menant J, Delbaere K, Lord SR, Pijnappels M (2010a) Consequences of lower extremity and trunk muscle fatigue on balance and functional tasks in older people: a systematic literature review. BMC Geriatr 10:56

Helbostad JL, Taraldsen K, Granbo R, Yardley L, Todd CJ, Sletvold $\mathrm{O}(2010 \mathrm{~b})$ Validation of the falls efficacy scale-international in fall-prone older persons. Age Ageing 39(2):259

Kemi OJ, Wisloff U (2010) High-intensity aerobic exercise training improves the heart in health and disease. J Cardiopulm Rehabil Prev 30(1):2-11

Kressig RW, Herrmann FR, Grandjean R, Michel JP, Beauchet O (2008) Gait variability while dual-tasking: fall predictor in older inpatients? Aging Clin Exp Res 20(2):123-130

Lepers R, Bigard AX, Diard JP, Gouteyron JF, Guezennec CY (1997) Posture control after prolonged exercise. Eur J Appl Physiol Occup Physiol 76(1):55-61

Lutz W, Sanderson W, Scherbov S (2008) The coming acceleration of global population ageing. Nature 451(7179):716-719

Middleton J, Sinclair P, Patton R (1999) Accuracy of centre of pressure measurement using a piezoelectric force platform. Clin Biomech (Bristol, Avon) 14(5):357-360

Midgley AW, McNaughton LR, Polman R, Marchant D (2007) Criteria for determination of maximal oxygen uptake: a brief critique and recommendations for future research. Sports Med 37(12): 1019-1028

Nardone A, Tarantola J, Giordano A, Schieppati M (1997) Fatigue effects on body balance. Electroencephalogr Clin Neurophysiol 105(4):309-320

Oja P, Laukkanen R, Pasanen M, Tyry T, Vuori I (1991) A 2-km walking test for assessing the cardiorespiratory fitness of healthy adults. Int J Sports Med 12(4):356-362

Owings TM, Grabiner MD (2003) Measuring step kinematic variability on an instrumented treadmill: how many steps are enough? J Biomech 36(8):1215-1218
Parijat P, Lockhart TE (2008) Effects of lower extremity muscle fatigue on the outcomes of slip-induced falls. Ergonomics 51(12):1873-1884

Peterson MJ, Pieper CF, Morey MC (2003) Accuracy of $V_{2}(\max )$ prediction equations in older adults. Med Sci Sports Exerc 35(1):145-149

Riskowski JL, Mikesky AE, Bahamonde RE, Alvey TV 3rd, Burr DB (2005) Proprioception, gait kinematics, and rate of loading during walking: are they related? J Musculoskelet Neuronal Interact 5(4):379-387

Rubenstein LZ (2006) Falls in older people: epidemiology, risk factors and strategies for prevention. Age Ageing 35(Suppl 2):37-41

Salavati M, Hadian MR, Mazaheri M, Negahban H, Ebrahimi I, Talebian S, Jafari AH, Sanjari MA, Sohani SM, Parnianpour M (2009) Test-retest reliability of center of pressure measures of postural stability during quiet standing in a group with musculoskeletal disorders consisting of low back pain, anterior cruciate ligament injury and functional ankle instability. Gait Posture 29(3):460-464

Schmid M, Conforto S, Bibbo D, D’Alessio T (2004) Respiration and postural sway: detection of phase synchronizations and interactions. Hum Mov Sci 23(2):105-119

Stevens JA, Corso PS, Finkelstein EA, Miller TR (2006) The costs of fatal and non-fatal falls among older adults. Inj Prev 12(5):290-295

Trombetti A, Hars M, Herrmann FR, Kressig RW, Ferrari S, Rizzoli $\mathrm{R}$ (2011) Effect of music-based multitask training on gait, balance, and fall risk in elderly people: a randomized controlled trial. Arch Intern Med 171(6):525-533 